\begin{tabular}{|c|l|}
\hline Title & $\begin{array}{l}\text { Chirality transfer from chiral solvents and its memory in an azobenzene derivative exhibiting photo-switchable } \\
\text { racemization }\end{array}$ \\
\hline Author(s) & Thomas, Reji; Tamaoki, Nobuyuki \\
\hline Citation & $\begin{array}{l}\text { Organic \& Biomolecular Chemistry, 9(15), 5389-5393 } \\
\text { https://doi.org/10.1039/010b05453h }\end{array}$ \\
\hline Issue Date & 2011-08-07 \\
\hline Doc URL & http://hdl.handle.net/2115/49100 \\
\hline Rights & Org. Biomol. Chem., 2011, 9,5389-5393- Reproduced by permission of The Royal Society of Chemistry (RSC) \\
\hline Type & article (author version) \\
\hline File Information & OBC9-15_5389-5393.pdf \\
\hline
\end{tabular}

Instructions for use 


\title{
Chirality Transfer from Chiral Solvents and its Memory in Azobenzene Derivative Exhibiting Photo-switchable Racemization
}

\author{
Reji Thomas $^{a}$ and Nobuyuki Tamaoki ${ }^{{ }}{ }^{*}$ \\ Received (in $X X X, X X X) X$ th $X X X X X X X X X 20 X X$, Accepted Xth $X X X X X X X X X 20 X X$ \\ s DOI: $10.1039 / \mathbf{b 0 0 0 0 0 0 x}$
}

The transfer and dynamic fixation of chirality in cyclic azobenzenes using $R-(+)-1-$ phenylethylalcohol $(R-$ PEA) and S-(-)-1-phenylethylalcohol (S-PEA) as solvents or additives are investigated. The cyclic azobenzenes used in this study carry 1,5-dioxynaphthalene moiety as rotating unit, connected to the photoisomerizing $(E-Z)$ azobenzene unit with spacers of varying lengths. With the suitable lengths of the 10 spacers the molecules exhibit stable enantiomers originated from the element of planar chirality in the $E$ form due to the stopped rotation of the rotor, while in the $Z$ form the allowed rotation results in racemization. The $C D$ spectra of racemic compounds in $E$ form in chiral solvents were inert or almost negligible before irradiation, while $366 \mathrm{~nm}$ irradiation causing $E-Z$ photoisomerization resulted in induction of clear $C D$ bands. The thermal or photochemical reverse Z-E isomerization causes a change in 15 the CD spectra to the new ones which are reasonably matching with the spectra of the pure enantiomers recorded in non-chiral solvents. The obtained new CD spectra are maintained even in a racemic solvent system attained by the dilution with equal amount of chiral solvent of opposite stereo structure. These results indicate that the chirality is transferred from the chiral solvents or additives to the racemizing $Z$ form of cyclic azobenzene and it is fixed in the non-racemizing $E$ form. The molecule without

20 racemization in both $E$ and $Z$ forms did not show any significant induced $C D$ bands irrespective of $E-Z$ isomerizations. The molecule showing racemization in $E$ and $Z$ form just show the non fixed induced CD. The property of the photo-switchable racemization is necessary for the effective transfer and temporal fixation of the chirality in this type of chirality sensors.

\section{Introduction}

${ }_{25}$ The induction, transfer, amplification and memory of chirality are extensively discussed topics in the various fields of science such as chemistry, biology, pharmacology and material science. ${ }^{1}$ The research in this area ranges from the basic research to explore the origin of homochiral structures in nature ${ }^{2}$ to the practical aspects 30 of chirality transfer which focuses on the enantio selective synthesis, ${ }^{3}$ sensing ${ }^{4}$ and molecular device applications. ${ }^{5}$ Concerning the molecular sensors for chirality, in particular the dynamic induction and memory of chirality in molecular structures or alignment are important. There are many works on 35 induced CD of organic molecules such as benzophenone ${ }^{6}$ or Zazobenzene $^{7}$ in chiral solvents. In these systems the chiral information is dynamically transferred from solvents to the molecules, but on removal of the chiral solvents the chiral information is disappeared. Yashima et al. demonstrated a new 40 system where the helicity is induced on a stereo regular cistransoidal poly((4-carboxyphenyl)acetylene) by an optically active chiral amine. ${ }^{\text {a }}$ This macromolecular system also showed a non-volatile memory effect of the induced helicity on removal of the chiral amine. However, the induced helical structure should
${ }_{45}$ be in an equilibrated state, since the secondary structure of the polymer, which is the origin of the chirality in this study, is stabilized just by weak intramolecular interactions. Hence the chiral memory based on secondary structure of the polymer is incomplete. In this perspective, small molecules with well ${ }_{50}$ defined enantiomeric structures and switchable racemization property are preferable for chiral sensors.

Azobenzene-based compounds received greater attention in the field of photo-switchable molecular systems attributed to the large structural change upon $E-Z$ isomerization. ${ }^{8,9}$ Recently, we 55 have demonstrated the dynamic racemization property in a newly synthesized cyclic azobenzene by photo switching of the on-off rotation of a naphthalene rotor. ${ }^{10}$ The stopped rotation of the 1,5substituted naphthalene in the macro cyclic structure is the origin of the planar chiral nature of this molecule. The rotation of the 60 naphthalene rotor viz. racemization is dynamically switched by changing the cavity size of the macrocycle achieved by the photoinduced $E-Z$ isomerization of the azobenzene unit.

In this study we apply aforesaid dynamically racemizing molecular system as a chirality sensor with the memory effect. ${ }_{65}$ The temporal chirality is transferred from the solvents or additives to the cyclic azobenzene in the $Z$ form and it is memorized in the $E$ form by $Z-E$ isomerization. 


\section{Experimental}

All the starting materials for the synthesis of the cyclic azobenzenes were obtained from commercial suppliers (TCI and Wako pure chemicals) and were used as obtained without further 5 purifications. The chiral solvents $R-(+)-1$-phenylethylalcohol $(R-$ PEA) and $S$-(-)-1-phenylethylalcohol (S-PEA) of $98 \%$ enantiomeric purity were purchased from Wako pure chemicals Ltd.

\section{Synthesis of cyclic azobenzenes ( 1 - 3)}

All the cyclic azobenzenes, presented in this study were synthesized using the previously reported procedures from our group. ${ }^{10}$ The cyclic azobenzenes (compound 1, 2 and 3) were synthesized by the reduction of corresponding dinitro compounds. The structure and purity of the compounds were assessed using 15 NMR spectroscopy (JEOL ECX 400 NMR spectrometer) and matrix assisted laser desorption/ionization time-of-flight mass spectrometry (MALDI TOF MS).

\section{UV-Vis and Circular Dichroism Spectroscopic Experiments}

20 The samples for the $\mathrm{CD}$ spectroscopic studies were prepared by dissolving the weighed quantity of the $E$ cyclic azobenzenes (racemic) in known volume of chiral solvents $R$-PEA or S-PEA. The absorption spectra of the solutions were measured on a single beam UV-Vis spectrometer illuminated with a diode array laser 25 and the CD spectra were recorded on JASCO J-720U spectropolarimeter. Photoisomerization studies were conducted on a super high-pressure mercury lamp (500W, USHIO Inc.) using appropriate filters (366 or $436 \mathrm{~nm}$ ). The measurements of absorption and $\mathrm{CD}$ spectra and the photoisomerization studies 30 were conducted in a cuvette with a 1-mm light path.

\section{Results and discussions}

Scheme 1 shows chemical structure of the cyclic azobenzene used in this study. Compound $\mathbf{1}$ has stable enantiomers due to the stopped rotation of the naphthalene moiety both in $E$ and $Z$ forms.

${ }_{35}$ Compound 3 shows the free rotation of the naphthalene rotor irrespective of structure of azobenzene. In contrast to this, compound 2 shows switchable rotation of the rotor moiety. The

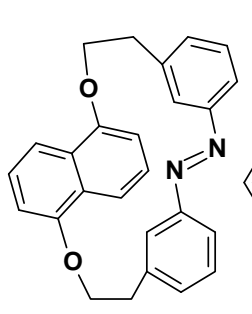

1

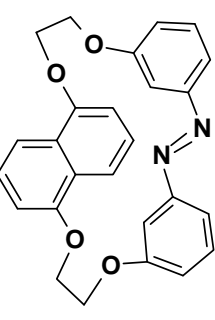

2

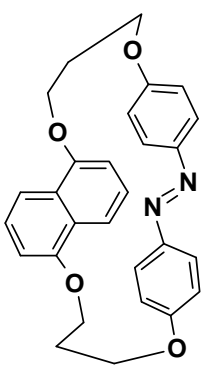

3
Scheme 1: Molecules presented in this study

40 rotation is completely hindered in $E$ form while it is allowed in $Z$ form, affording the photo-switchable racemization in this molecule. These observations can be explained by the difference in the cavity size of the macrocycles tuned by the spacer length and the $E-Z$ isomerization of the azobenzene part. UV-visible 45 spectrum for the dilute solution of racemic $E-\mathbf{1}$ in chiral solvent $R$-PEA showed similar characteristics of $E$ azobenzenes with absorption maxima at $302 \mathrm{~nm}$ corresponding to the $\pi-\pi^{*}$ transitions and at $450 \mathrm{~nm}$ due to the $n-\pi^{*}$ transitions of the azobenzene in addition to the structured features assignable to 50 naphthyl group around $300 \mathrm{~nm}$ (see supporting information Fig. S1a), which are similar to those in non chiral solvents. ${ }^{5 a}$

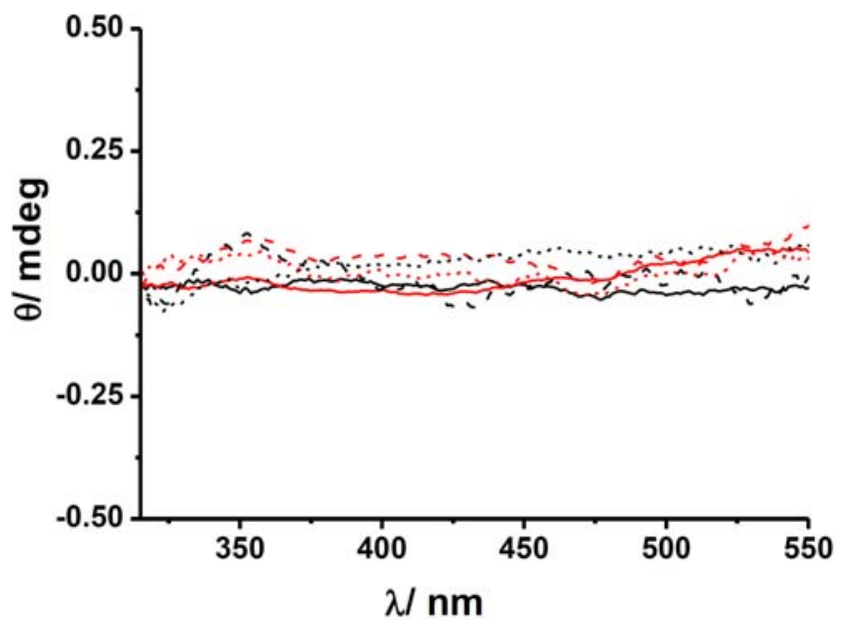

Fig. 1 CD Spectra of the racemic mixture of compound 1 in chiral solvents R-PEA (black) and S-PEA (red) (a) before irradiation (solid), (b) 55 after irradiation with $366 \mathrm{~nm}$ (dashed) and (c) irradiated with $436 \mathrm{~nm}$ (short dash). Solution concentration: $4.6 \times 10^{-4} \mathrm{M}$.

The CD spectra of the racemic $E-\mathbf{1}$ in chiral solvent $R$ - and $S$ PEA are shown in Fig. 1. The spectra do not exhibit any significant bands in both the solvents. Even after irradiation with ${ }_{60} 366 \mathrm{~nm}\left(\mathrm{PSS}_{366}\right)$ and subsequent irradiation with $436 \mathrm{~nm}$ light featureless CD spectra were retained. A dilute solution of $\mathbf{3}$ in both the chiral solvents $R$ - and S-PEA exhibited UV-Vis absorption bands with maxima at 300, 360 and $450 \mathrm{~nm}$ (supporting information Fig. S1c). The CD spectra for a solution

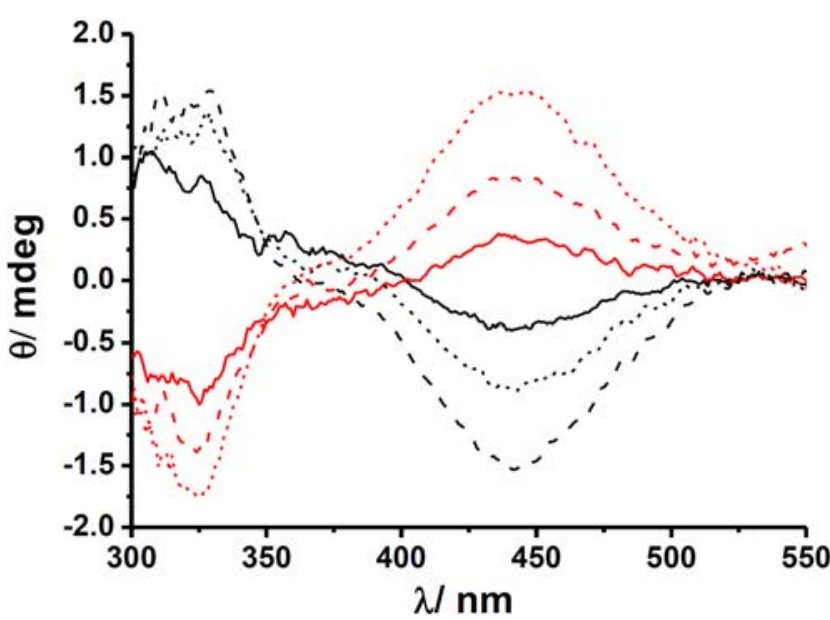

Fig. 2 CD spectra for the racemic mixture of the compound 3 in chiral solvent $R$-PEA (black) and $S$-PEA (red) (a) as prepared (solid), (b) after $366 \mathrm{~nm}$ light irradiation, (dashed) and (c) after $436 \mathrm{~nm}$ irradiation (short dash). Solution concentration: $7.73 \times 10^{-4} \mathrm{M}$.

70 in R-PEA showed relatively intense bands with positive $(330 \mathrm{~nm}$ and $370 \mathrm{~nm}$ ) and negative $(442 \mathrm{~nm})$ signs (Fig. 2). After irradiation with $366 \mathrm{~nm}$ a significant increase in the intensity of 
the band at $442 \mathrm{~nm}$ was observed. It is noteworthy that the positive band observed at $370 \mathrm{~nm}$ is completely disappeared on irradiation of $366 \mathrm{~nm}$ light, while the band at $330 \mathrm{~nm}$ showed a small increase in intensity. The CD spectrum recorded after 436 $5 \mathrm{~nm}$ irradiation was the intermediate of those before and after 366 $\mathrm{nm}$ irradiation. The complete thermal Z-E isomerisation brought the $\mathrm{CD}$ spectra to the initial one.

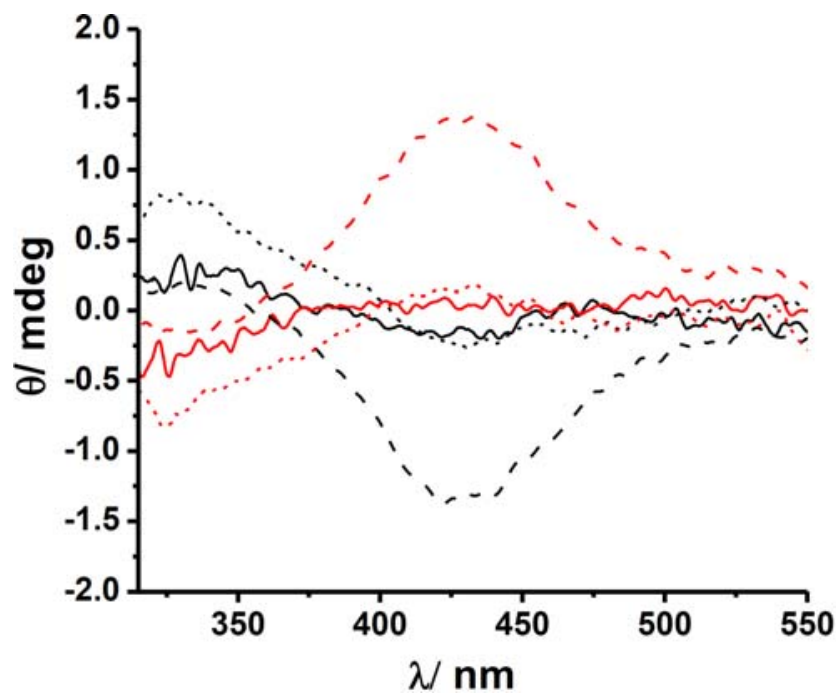

Fig. $3 \mathrm{CD}$ spectra for the racemic mixture of the compound $\mathbf{2}$ in chiral 10 solvent $R$-PEA (black) and S-PEA (red) (a) as prepared (solid), (b) after $366 \mathrm{~nm}$ light irradiation, (dashed) (c) after Z-E isomerization by keeping in dark at $25^{\circ} \mathrm{C}$ for 72 hours (short dash). Solution concentration $9.1 \times 10^{-4}$ M.

A similar experiment with compound 2 in chiral solvents $R$ 15 PEA and S-PEA were carried out. The absorption spectra of the dilute solutions of $\mathbf{2}$ in chiral solvents $R$-PEA and $S$-PEA showed absorption bands with $\lambda_{\max }$ at $297 \mathrm{~nm}$ and $440 \mathrm{~nm}$ (see Fig. S1b) comparable to the band structure in non-chiral solvents. ${ }^{10}$ The CD spectrum of 2 in $R$-PEA showed a weak positive band centered 20 around $327 \mathrm{~nm}$ along with a weak negative band at $428 \mathrm{~nm}$ (see Fig. 3). A mirror image spectrum was obtained for $E-2$ (racemic) in $S$-PEA. The exposure of $\mathbf{2}$ in $R$-PEA to $366 \mathrm{~nm}$ light resulted in the $E-Z$ isomerisation. The UV-vis absorption spectrum showed a decrease in the intensity of the $\pi-\pi^{*}$ transition band $(297 \mathrm{~nm})$ of 25 azobenzene chromophore along with a small increment in the intensity of the band at $440 \mathrm{~nm}\left(\mathrm{n}-\pi^{*}\right.$ transition) (see Fig. S1b). The corresponding CD spectrum showed a significant change with the formation of a strong negative band at $428 \mathrm{~nm}$ accompanied by a weaker positive band at $327 \mathrm{~nm}$. In contrast to 30 compound 3 a complete thermal Z-E isomerization of $\mathbf{2}$ in chiral solvent did not bring the CD spectra of the solution to the initial one, but to the new spectrum with an intense band at $327 \mathrm{~nm}$ and a weak band at $428 \mathrm{~nm}$ (See Fig. 3). A mirror image spectrum was obtained with the solution of 2 in S-PEA. The features of the

${ }_{35} \mathrm{CD}$ spectra obtained after thermal isomerization completely matches with those of the pure enantiomers of E-2 in non-chiral solvents. ${ }^{10}$ The origin of a peak at $327 \mathrm{~nm}$ along with a small band at $428 \mathrm{~nm}$ may be due to the enrichment of one of the stable chiral structure (enantiomer) in the $E$ - state.

40 To prove the role of chiral solvent in chiral induction of cyclic azobenzenes we have performed an experiment where the chiral solvents used as additive in dichloromethane solutions of $\mathbf{2}$ (see
Fig. 5). The CD spectra recorded for dichloromethane solutions before and after irradiation $(366 \mathrm{~nm})$ were silent in nature (see

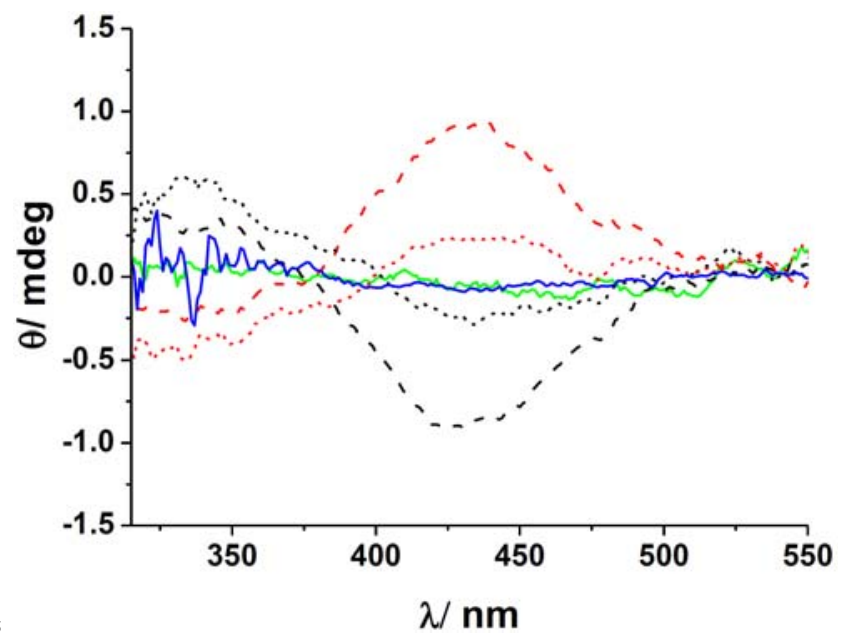

Fig. 4 Chiral solvents as additives to a dichloromethane solution of 2: CD spectra of (a) 2 in dichloromethane (blue), (b) after 366 irradiation (green) (c) on addition of chiral solvent to the 366 irradiated solution (dashed curves) (d) after reverse (Z-E) isomerization by $436 \mathrm{~nm}$ irradiation (short dash). Solution concentration: $1.7 \times 10^{-3} \mathrm{M}$.

blue and green curves) whereas, that recorded after the addition of an equal volume of chiral solvent to the $366 \mathrm{~nm}$ irradiated solution of $\mathbf{2}$ in dichloromethane (dashed curves) showed significant bands. The band at $428 \mathrm{~nm}$ of the spectra clearly ${ }_{55}$ indicates the influence of the chiral solvent on Z- $\mathbf{2}$ in modifying the naphthalene rotor to a favored orientation which in turn results in a chiral structure. The photo controlled chirality transfer and the memory of chiral structure were confirmed by the irradiation of the solution under $436 \mathrm{~nm}$ light which gives an $E$ 60 and $Z$ isomer ratio of $67: 33$. The $C D$ spectra recorded for the solution after the irradiation with $436 \mathrm{~nm}$ light showed a change in the intensity ratio of the spectra with a decrease in intensity at $428 \mathrm{~nm}$ with a concomitant increase in intensity at $327 \mathrm{~nm}$. The changes in the spectral features once again confirm that the chiral ${ }_{65}$ solvent induces preferred orientation the structure in $Z$ states of

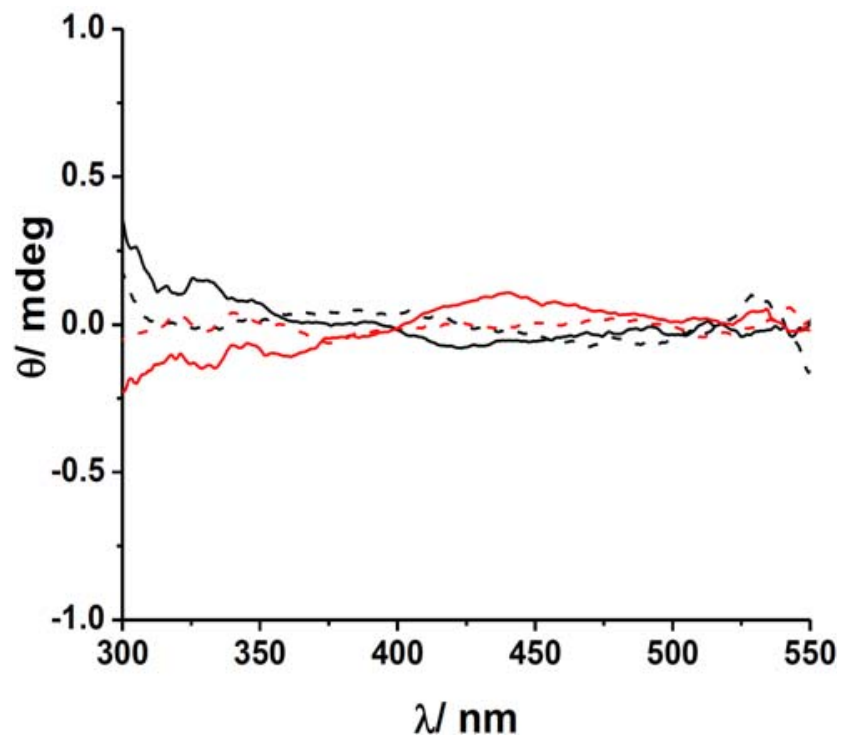


Fig. $5 \mathrm{CD}$ spectra for the racemic mixture of the compound 2 in chiral solvent $R$-PEA (black) and S-PEA (red) (a) as prepared (solid) (b) after addition of the equal amount of chiral solvent with opposite sterio structure (dashed). Solution concentration: $9.45 \times 10^{-4} \mathrm{M}$.

5 the 2 and $Z-E$ isomerisation fixes the chiral structure in $E$ state.

In order to confirm the origin of CD band at $428 \mathrm{~nm}$ and 327 $\mathrm{nm}$ observed on thermal $Z-E$ reverse isomerization, we have performed an experiment by mixing chiral solvents, where the chiral solvent $S$-PEA is added to a solution of 2 in $R$-PEA or vice

10 versa. Fig. 5 shows the CD spectra recorded for a solvent mixing experiment conducted for $\mathbf{2}$ before irradiation. The spectra of $\mathbf{2}$ in $R$-PEA showed small induced CD signal at $327 \mathrm{~nm}$ and $428 \mathrm{~nm}$, while the addition of equal amount of $S$-PEA to this solution resulted in a featureless $\mathrm{CD}$ spectra. A similar observation can be 15 made in an experiment, where an equal amount of $R$-PEA is added to a solution of $\mathbf{2}$ in S-PEA.

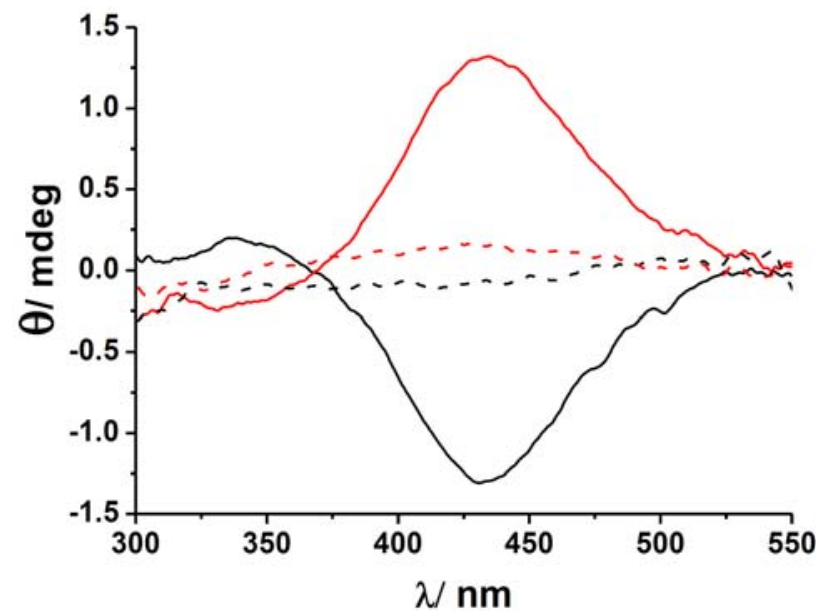

Fig. $6 \mathrm{CD}$ spectra for the racemic mixture of the compound 2 in chiral solvent $R$-PEA (black) and $S$-PEA (red) (a) after $366 \mathrm{~nm}$ irradiation

(solid) (b) after addition of the equal amount of chiral solvent with opposite sterio structure (dashed). Solution concentration: $9.45 \times 10^{-4} \mathrm{M}$.

Consequently, a solvent mixing experiment with solutions of $\mathbf{2}$ in chiral solvents after $366 \mathrm{~nm}$ irradiation showed a diminished intensity at $327 \mathrm{~nm}$ and $428 \mathrm{~nm}$ indicating these bands are clearly

${ }_{25}$ due to induced CD signal of Z-2 in chiral solvents (Fig. 6). In a similar experiment, we added the solvent of opposite chiral structure to the solutions of $E-2$ formed by reverse isomerization

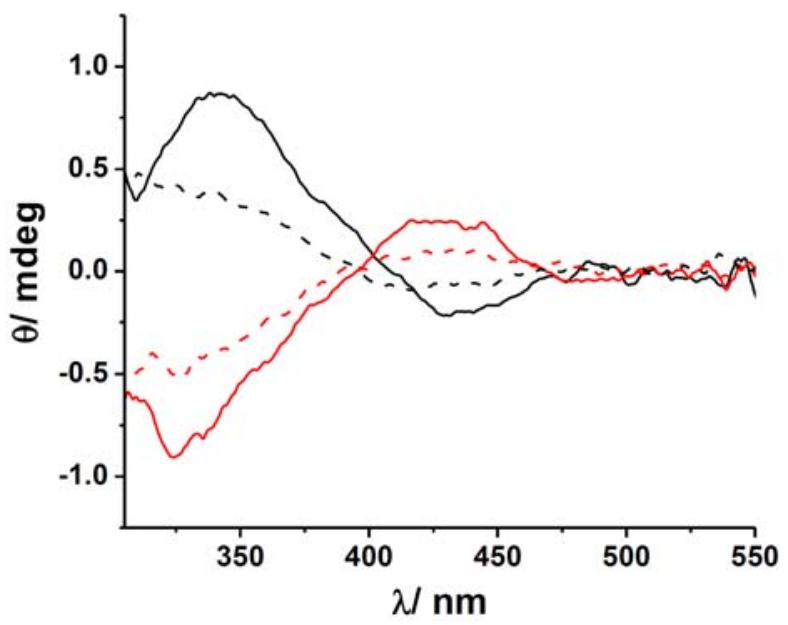

Fig. $7 \mathrm{CD}$ spectra for the racemic mixture of the compound 2 in chiral 30 solvent $R$-PEA (black curves) and $S$-PEA (red curves) (a) after thermal Z$E$ isomerization (solid) (b) after addition of the equal amount of chiral solvent of opposite chirality (dashed). Solution concentration: $9.45 \times 10^{-4}$ M.

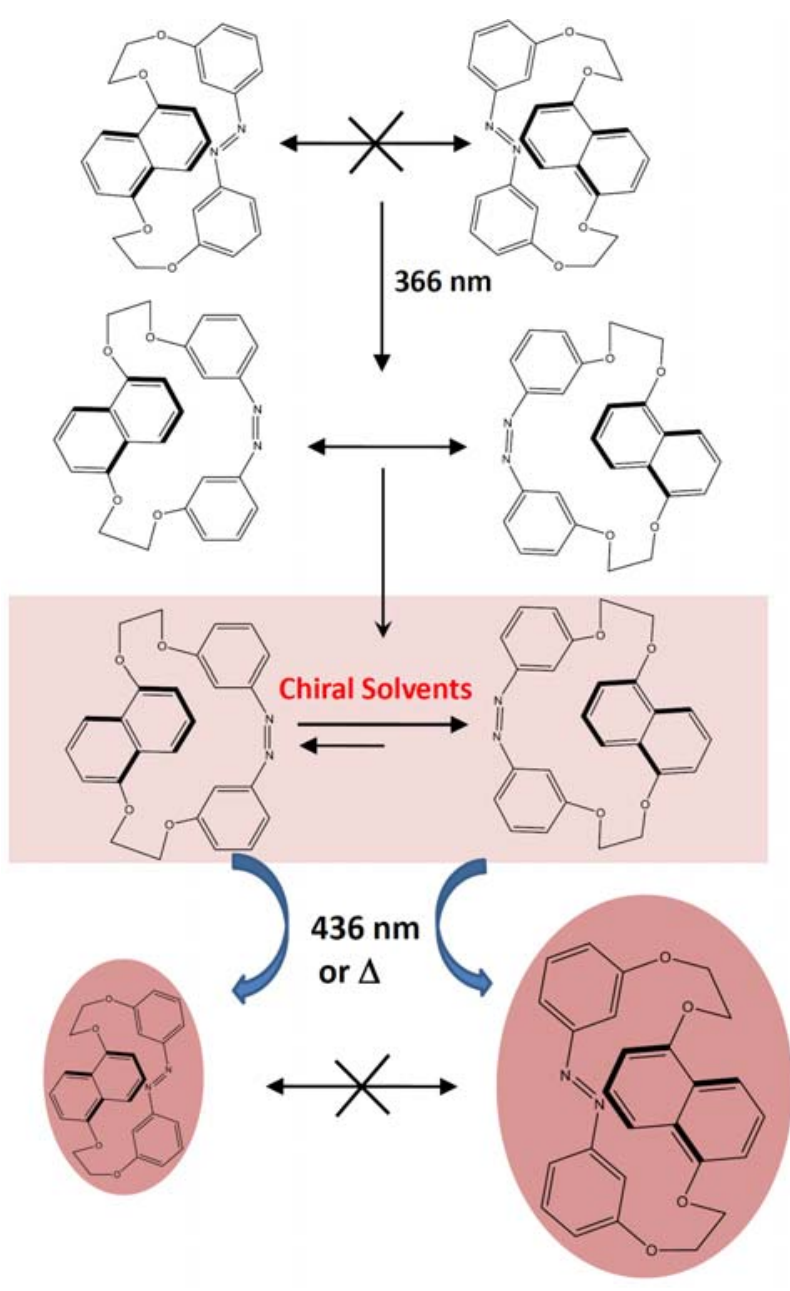

Scheme 2. Photochemical and thermal processes involved in chiral sensing of molecule 2 , in $R$-PEA and $S$-PEA.

from the photochemically formed $Z$ state. In contrast to the 40 previous solvent mixing experiments the $\mathrm{CD}$ spectra recorded for this solution retained the signal at $327 \mathrm{~nm}$ and $428 \mathrm{~nm}$ but with reduction in intensity into half due to the dilution (Fig. 7). This result suggests that the observed CD signal obtained after thermal reverse isomerization from the photochemically formed $Z$ state is 45 due to the formation of stable enantiomeric excess.

We have estimated the extent of fixation of the transferred chirality (memory) from chiral solvents to the cyclic azobenzene 2 by a quantitative analysis of CD spectra. The comparison of the $\mathrm{CD}$ band intensities at $327 \mathrm{~nm}$ for compound 2 with that of its 50 pure enantiomers in same racemic mixture of chiral solvents showed a chiral enrichment of $1.5 \%$ respectively (CD spectra of authentic pure enantiomers are shown in Fig. S2). The chiral HPLC analysis of the pure enantiomers in chiral solvents showed that these enantiomers are stable over a week (see Fig. S3). Thus, 55 we could achieve the photo fixation of the transferred chirality in 
cyclic azobenzene with rendered $(E)$ and allowed rotations $(Z)$.

Scheme 2 depicts the plausible mechanism of chiral sensing of the cyclic azobenzene 2 in chiral solvents $R$-PEA and S-PEA. The chiral solvent favors one of the enantiomeric structures of $\mathbf{2}$ in $Z$ 5 state by the orientation of naphthalene rotor which in turn results in a shift of the enantiomeric equilibrium. The $\mathrm{CD}$ signal observed from the solution of Z-2 in chiral solvents is clearly an induced signal as evident from the solvent mixing experiment. The sustained CD signal observed for E-2 after thermal Z-E 10 isomerization even in the presence chiral solvent of opposite chiral structure substantiate the formation of fix chiral structure of 2. A similar but permenant formation of chiral structure has been studied previously in the case of electrochemical pinacolization ${ }^{11}$ and photochemical reactions with helicenes in ${ }_{15}$ chiral solvents ${ }^{12}$ While, the present study demonstrate a dynamic fixation of transferred chirality sensor which is controlled by simple $E-Z$ photoisomerizations.

\section{Conclusion}

In summary, we demonstrated the transfer and dynamic fixation 20 of the chirality in a cyclic azobenzene with napthalene rotor in chiral solvents. In contrast to the previously reported polymeric systems where the chirality is fixed as an indefinite secondary structure, here in molecule 2 , the transferred chirality is memorized as a well defined planar chirality of the single small 25 molecule.

\section{Notes and references}

${ }^{a}$ Research Institute for electronic Science, Hokkaido University, Kita-Ku, N-20, W-10, Sapporo, 001-0020, Japan. Fax: +81 11706 9357; Tel: +81 11706 9356;

30 E-mail: tamaoki@es.hokudai.ac.jp

$\uparrow$ Electronic Supplementary Information (ESI) available: Uv-vis spectra of compounds 1, 2 and $\mathbf{3}$ in chiral solvents, CD spectra of enatiomers of $\mathbf{2}$ in chiral solvents and enantiomeric stability of $\mathbf{2}$ in chiral solvents monitored by chiral HPLC. See DOI: 10.1039/b000000x/

35 1. (a) W. J. Lough and I. W. Wainer, Chirality in Nature and Applied Science, CRC Press. Oxford, 2002; (b) N. Berova, K. Nakanishi and R. W. Woody, Circular Dichroism Principles and Applications, Wiley-VCH, New York, $2^{\text {nd }}$ edn. 2000; (c) E. Francotte, W. Lindner, R. Mannhold, H. Kubinyi and G. Folkers, Chirality in Drug Research

40 -Methods and Principles in Medicinal Chemistry, Wiley-VCH; 1 edn., 2007.

2. (a) W. A. Bonner, Top. Steriochem. 1988, 18, 1; (b) A. S. Garay, Nature, 1968, 219, 338. (c) K. Keosian, The Orgin of life, $2^{\text {nd }}$ edn., Reinhold, New York, 1968; (d) S. Mason, Chem. Soc. Rev. 1988, 17, 347; (e) S. Kojo, Symmetry 2010, 2, 1022.

3. R. Noyori and S. Hashiguchi, Acc. Chem. Res. 1997, 30, 97.

4. (a) E. Yashima, T. Matsushima and Y. Okamoto, J. Am. Chem. Soc, 1997,119, 6345. (b) D. Pijper and B. L. Feringa, Angew. Chem. Int. Ed. 2007, 46, 3693. (c) E. Yashima, K. Maeda and Y. Okamoto, Nature, 1999, 399, 449. (d) G. Wulff, H. Schmidt, H. Witt and R. Zentel, Angew. Chem. Int. Ed. 1994, 33, 188. (e) T. Miyagawa, A. Furuko, K. Maeda, H. Katagiri, Y. Furusho and E. Yashima, J. Am. Chem. Soc. 2005, 127, 5018. (f) E. Yashima, K. Maeda, T. Nishimura, Chem. Eur. J., 2004, 10, 42. (g) M. Wolffs, S. J. George, Z. Tomovic,

55 S. C. J. Meskers, A. P. H. J Schenning, E. W. Meijer Angew. Chem. Int. Ed.2007, 46, 8203.

5. (a) Mathews, M.; Tamaoki, N. J. Am. Chem. Soc. 2008, 130, 11409. (b) Tamaoki, N. Adv. Mater. 2001, 13, 1135. (c) Zhou, Y. ; Zhang, D.; Zhu, L.; Shuai, Z.; Zhu, D. J. Org. Chem. 2006, 71, 2123 (d) 60 Akiyama, H.; Mallia, V. A.; Tamaoki, N. Adv. Funct. Mater. 2006, 16, 477. (e) Q. Li, L. Green, N. Venkataraman, I. Shiyanovskaya, A.
Khan, A. Urbas and J. W. Doane, J. Am. Chem. Soc. 2007, 129, 12908. (f) T. J. White, R. L. Bricker, L. V. Natarajan, N. V. Tabiryan, L. Green, Q. Li, T. J. Bunning, Adv. Funct. Mater. 2009, 19, 1.

65 6. (a) B. Bosnich, J. Am. Chem. Soc., 1967, 89, 6143(b) P. E. Schipper, J. M. O'Brien and D. D. Ridley, J. Phys. Chem. 1985, 89, 5805.

7. L. D. Hayward, R. N. Totty, Can. J. Chem., 1971, 49, 624.

8. (a) C. Dugave and L. Demange, Chem Rev. 2003, 103, 2475. (b) S. Shinkai, T. Nakaji, Y. Nishida, T. Ogawa and O. Manabe, J. Am. 70 Chem. Soc. 1980, 102, 5860. (c) A. Dawn, N. Fujita, S. Haraguchi, K. Sada and S. Shinkai, Chem. Commun. 2009,1356.

9. (c) R. H. El Halabieth, O. Mermut and C. Barrett, J. Pure Appl. Chem. 2004, 76, 145. (d) H. Akiyama, V. A. Mallia and N. Tamaoki, Adv. Funct. Mater. 2006, 16, 477. (e) M. Han, D. Ishikawa, T. Honda, E. 75 Ito and M. Hara, Chem. Commun., 2010, 46, 3598. (f) T. Muraoka, K Kinbara, Y. Kobayashi and T. Aida, J. Am. Chem. Soc. 2003, 125, 5612. (g) T. Muraoka, K. Kinbara and, T. Aida, Nature 2006, 440, 512.(h) S. Muramatsu, K. Kinbara, H. Taguchi, N. Ishii and T. Aida, J. Am. Chem. Soc., 2006, $128,3764$.

80 10. M. C. Basheer, Y. Oka, M. Mathews and N. Tamaoki, Chem. Eur. J. 2010, 16, 3489.

11. D. Seebach and H. A. Oeil, Angew. Chem. Int. Edn. 1975, 14, 634

12. W. H. Laarhoven, T. J. H. M. Cuppen, J. Chem. Soc. Perkin. II, 1978, 315 .

This journal is (C) The Royal Society of Chemistry [year]

Journal Name, [year], [vol], 00-00 | 5 
Cite this: DOI: 10.1039/c0xx00000x

Table of Contents Entry

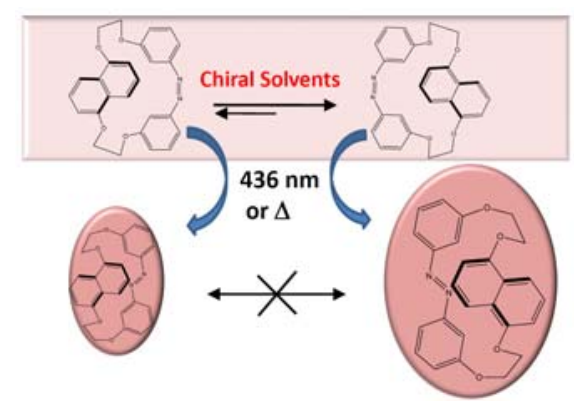

The chirality is trasferred form a chiral solvent and memorized as 5 a well defined planar chirality of the single small molecule. 